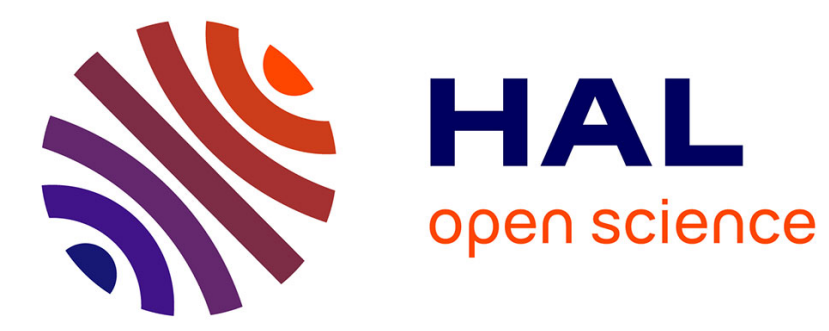

\title{
SURFACE AND INTERFACE MAGNETISM BY MÖSSBAUER SPECTROSCOPY
}

\author{
T. Shinjo
}

\section{To cite this version:}

T. Shinjo. SURFACE AND INTERFACE MAGNETISM BY MÖSSBAUER SPECTROSCOPY. Journal de Physique Colloques, 1979, 40 (C2), pp.C2-63-C2-68. 10.1051/jphyscol:1979222 . jpa00218600

\section{HAL Id: jpa-00218600 https://hal.science/jpa-00218600}

Submitted on 1 Jan 1979

HAL is a multi-disciplinary open access archive for the deposit and dissemination of scientific research documents, whether they are published or not. The documents may come from teaching and research institutions in France or abroad, or from public or private research centers.
L'archive ouverte pluridisciplinaire HAL, est destinée au dépôt et à la diffusion de documents scientifiques de niveau recherche, publiés ou non, émanant des établissements d'enseignement et de recherche français ou étrangers, des laboratoires publics ou privés. 


\title{
SURFACE AND INTERFACE MAGNETISM BY MÖSSBAUER SPECTROSCOPY
}

\author{
T. Shinjo \\ Institute for Chemical Research, Kyoto University, Uji, Kyoto-fu, 611, Japan
}

Résumé.- Le comportement magnétique d'un cristal ordonné magnétiquement, près d'une surface ou $d^{\prime} u n$ interface est un phénomène très intéressant. Le moment magnétique local d'un atome superficiel, $1^{\prime}$ influence de la tempêrature sur 1'aimantation de surface et $I^{\prime}$ anisotropie superficielle ont été étudiés par effet Mössbauer du ${ }^{57} \mathrm{Fe}$. Les résultats obtenus par d'autres méthodes sont analysés brièvement. Les avantages et les limites de la spectrométrie Mössbauer pour l'étude des surfaces sont discutês.

Abstract.- Magnetic behaviors near a surface, or an interface, of a magnetically ordered crystal are of great interest. Local magnetic moment of a surface atom, temperature dependence of surface magnetization and surface anisotropy are studied from ${ }^{57} \mathrm{Fe}$ Mössbauer measurements. The results obtained by complementary means are briefly reviewed. The advantages and also limits of the Mössbauer spectroscopy as a tool for surface magnetic studies are discussed.

\section{Problems in surface magnetism. - In recent years} magnetic properties of transition metal surfaces have been of great interest from a viewpoint of fundamental physics and also in connection with surface chemistry such as catalysis research. Magnetic behaviors near a surface or an interface in a magnetically ordered crystal may differ in many respects from those of the inside. For an understanding of the perturbation of magnetic order at a surface boundary, not only clean vacuum surfaces but also various kinds of interfaces are of importance.

The main problems to be considered here are local magnetic moment, the temperature dependence of surface magnetization and surface anisotropy. Before describing the information obtained from Mössbauer spectroscopy, experimental results by complementary methods will be briefly surveyed.

1.1. Local magnetic moment.- In the metallic case, a local magnetic moment sensitively depends on the local environment. First of a11, the local magnetic moment of a surface atom at $\mathrm{T}=\mathrm{OK}$ must be made clear. Most studies on the surface magnetism have been done by using thin film samples and numerous papers have been published. The study of thin film magnetism has a rather long history but until recently the sensitivity of the measurements and the quality of the samples were not good enough to derive any significant information on surface magnetism.

Liebermann et al. have measured the magnetization of electrodeposited thin films of Fe, Co and $\mathrm{Ni}$ as a function of thickness and proposed the "dead layer model" to explain their results : namely the first surface layer has no magnetic moment $/ 1,2 /$. This conclusion is not generally believed but the term, dead layers, has been popularly used.

An investigation on epitaxial ferromagnetic thin films was reported by Gradmann /3/. By vacuum ceposition, very thin epitaxial films of $\mathrm{Fe}-\mathrm{Ni}$ alloy and Co, on Cu substrates, were prepared and the magnetization was measured by a sensitive torsion magnetometer. When the film was coated by $\mathrm{Cu}$ or $\mathrm{Ag}$, the magnetization of the thin film was actually the same as the bulk value but when coated by $\mathrm{Mn}$ or $\mathrm{C}$, a reduction of the magnetization was observed. The results suggest that the interface magnetic effect depends on the coating substance.

Koepke and Bergmann measured an anomalous Ha1l effect of very thin Fe films /4/. Their samples were vacuum deposited Fe films on cold substrates of amorphous alloys $(\mathrm{Pb}-\mathrm{Bi}$ or $\mathrm{Sn}-\mathrm{Cu})$. The magnetization of the films estimated for the Hall resistance was the same as the bulk value and it was concluded that the surface effect on the local magnetic moment is negligible.

A new technique to approach the interface magnetism was recently developed by neutron spectroscopists /5/. Sato and Abe measured the Bragg reflections of a multilayer sandwich film consisting of $20 \AA \mathrm{Fe}$ and $40 \AA$ Sio layers, and estimated the magnetic anomaly at the interface, if any, to be less than 1/10 of one atomic layer's magnetization.

It seems that a remarkable surface effect on the local magnetic moment only occurs at the topmost layer, or extends at most to a few surface 
layers. In order to catch a surface anomaly by thin film measurements, the thickness of the sample has to be extremely thin. Not only the sensitivity of the measurements but also high accuracy in thickness determination is usually necessary. In addition to surface effects, various kinds of size effects may be included in the results. It is desirable to study a surface of bulk specimen. From this point of view, Mössbauer spectroscopy using ${ }^{57} \mathrm{Co}$ or ${ }^{57} \mathrm{Fe}$ as a microprobe on a surface is a useful method, as will be described below.

Newly developed experimental techniques which can observe magnetic properties of clean vacuum surfaces of bulk crystals are the polarization measurements of photoemitted, field emitted and tunneling electrons $/ 6 /$. The electron spin polarization at a Ni surface was determined through electron capture by small angle refrected deuterons $/ 7 /$. These measurements only detect the electrons near the surfaces. It is however very difficult to separate a surface information from the bulk properties since the mechanism of electron emission is complicated.

Theoretical works on local magnetic moments at a surface site have been published only recently. In most cases, calculations were based on a tightbinding model assuming a free surface of crystallographically simple plane.

It is questionable to compare them quantitatively with experimental results on actual interfaces. However it is of interest to note the variety of the theoretical predictions.

Liebsch et al. showed the possibility of dead layers 18/. Fulde et a1. suggested the existence of an antife:-romagnetic surface layer $/ 9 /$. According to Desjonquerers and Cyrot-Lackmann, the surface magnetic moments of $\mathrm{Fe}$ and $\mathrm{Ni}$ are nearly the same as the bulk values $/ 10 /$. On the other hand, Teraoka and Kanamori suggested the enhancement of surface magnetic moment $/ 11 /$.

An interesting theoretical prediction is the existence of "live layers" in the surface of $\mathrm{Cr}$ or $\mathrm{V} / 11-13 /$. The possibility of local magnetic moment induced on 1 y at the surface has been discussed recently. At the moment, however, no Mössbauer experiment is relevant to this problem.

\subsection{Temperature dependence of surface magnetiza-}

tion.- Because the number of magnetic neighbours is reduced at a surface site, the Weiss field is generally expected to be smaller than the inside. Therefore even when the surface magnetic moment is not changed, the temperature dependence of the local magnetization at the surface may be different from the bulk curve. Many calculations have been carried out for the cases of thin films and also surface layers, on condition that all the local magnetic moment at $T=O K$ is the same even at the surface boundary. An example quated here as figure 1 is a part of the computor works by Binder and Hohenberg $/ 14 /$. It was suggested that the reduction of surface magnetization of this kind is only appreciable at relatively high temperatures. When the temperature approaches ${ }^{T}$, the surface effect extends more deeply into the crystal.

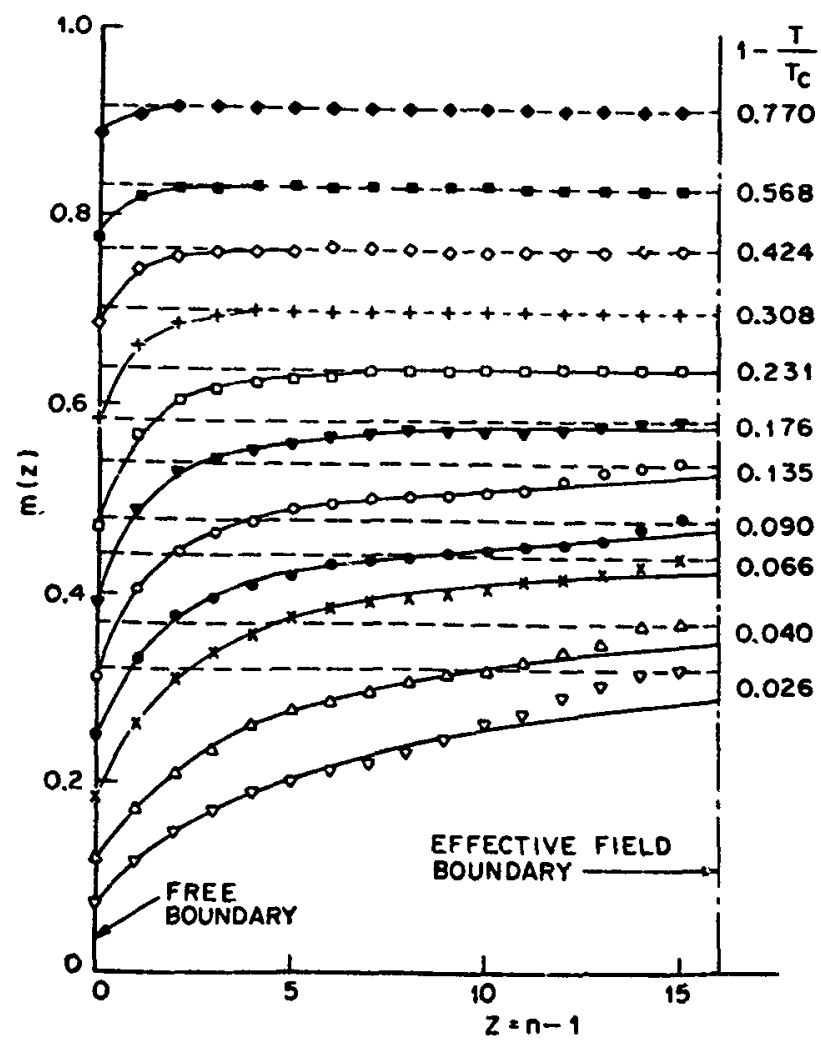

Fig. 1 : Monte Carlo calculation for the magnetization in a Heisenberg system by Binder and Hohenberg /15/. The surface is (100) of a simple cubic lattice. The effective field boundary condition at $\mathrm{n}=16$ layers forces the magnetization to the bulk value.

Concerning the temperature dependence of magnetization in a finite dimension, experimental studies are not fully following up the theoretical predictions. The temperature dependence of the magnetization of a very thin film, over the whole temperature range, is not possible to measure because of superparamagnetism and also because of chemical instability.

The temperature dependence of the surface 
magnetization of a bulk crystal can be measured from the observation of superlattice line of low energy electron diffraction. The measurement near $T_{C}$ on Nio crystal has been reported by Palmberg et al. $/ 15 /$ and 1ately by Namikawa /16/. Unfortunately this method is only applicable to an antiferromagnet.

1.3. Surface anisotropy.- In addition to the understanding of the local magnetic moment at a surface atom, the direction of the surface spin is of interest. A magnetic spin at a surface site has a lower symmetry than in the bulk and anisotropic interactions, which may be canceled by symetry in the interior need not to vanish at the surface. One may assume that a surface atom has a symmetry axis normal to the surface and therefore a strong anisotropy can exist perpendicularly to the surface plane. Such a surface anisotropy only exists at the topmost layers. As it is well known, the magnetization of a ferromagnetic film is oriented parallel to the plane due to the shape anisotropy. The energy of shape anisotropy and also the energy of crystalline anisotropy, per unit area of the film, decrease with a decrease of thickness. On the other hand, the surface anisotropy per unit area remains constant. In extremely thin films, by the surface anisotropy, the magnetization may be oriented normal to the film plane.

Gradmann firstly found the perpendicular magnetization of very thin epitaxial films of Fe-Ni alloy and Co when the thickness was as small as a few $\AA / 3 /$. Although the concept of surface anisotropy has sometimes been implicitely taken in the discussion of thin film magnetism, the existence of surface anisotropy is clearly evidenced on1y by the perpendicular magnetization, which was observed by Gradmann's measurements and Mössbauer spectroscopy as will be shown below.

Very recently the surface anisotropy in the case of $\mathrm{Ni}$ was theoretically treated by Takayama et al.

$/ 17 /$. A quantitative discussion of surface anisotropy is very difficult especially in the metallic case since a detailed knowledge on the electronic structure of the surface atom is necessary.

2. Mössbauer spectroscopic studies.-

2.1. Sourccesspectrososcony.- The minimum activity of ${ }^{57} \mathrm{Co}$ required for Mössbauer sources is in the order of $10 \mu \mathrm{Ci}$, whose amount is much less than one monatomic layer in an area of $1 \mathrm{~cm}^{2}$. If ${ }^{57} \mathrm{Co}$ atoms are uniformly deposited on a crystal surface, the Mössbauer emission spectra will give us information on the top surface layer of a bulk crystal. The commercial carrier-free ${ }^{57} \mathrm{Co}$ solution has a sufficiently high purity for this purpose. In metallic cases, the emission spectroscopy always observes the stable electronic state of ${ }^{57} \mathrm{Fe}$ atom and the emission spectra is essentially the same as the absorption spectra. The problem actually is : how to deposit the tracer amount of ${ }^{57} \mathrm{Co}$ on a wel1-defined surface.

The author's group already published some results on the surfaces of $\mathrm{Fe}$ and Co where the ${ }^{57} \mathrm{Co}$ source atoms were deposited by electrolytic means. On an Fe plate, natural Fe layers were electrolytically deposited in advance and successively ${ }^{57} \mathrm{Co}$ was deposited on the surface /18/. After finishing the deposition, the sample was cooled rapidly by liquid nitrogen. The Mössbauer spectra at $4.2 \mathrm{~K}$ showed that the state of ${ }^{57} \mathrm{Fe}$ atoms was metallic and ferromagnetic. The absence of any paramagnetic or oxidized fraction was certified also from the spectra. The hyperfine field was fairly distributed and the average $(290 \mathrm{k0e}$ ) was smaller than the bulk value (340 k0e). The intensity ratio of the six lines was nearly $3: 4: 1: 1: 4: 3$, which indicates that the magnetic spins are oriented parallel to the surface plane. No significant temperature dependence was observed between liquid nitrogen and liquid helium temperatures. The sample was not stable above liquid nitrogen temperature.

Similar results were obtained for ${ }^{57} \mathrm{Co}$ on a surface of bulk co (hep structure) and also on a surface of a Co film deposited on a Cu substrate (fcc structure) $/ 19 /$. The surfaces in these measurements were inevitably covered by ice, however, the state of $\mathrm{Fe}$ was always ferromagnetic and no dead layer was observed. It should be noted that the films of Liebermann et al. who claimed the existence of dead layers, also were electrolytically prepared.

Vacuum depositions of ${ }^{57} \mathrm{Co}$ were attempted by Burton and Godwin to study the behavior of Fe impurities on the surfaces of $\mathrm{W}$ and $\mathrm{Ag} / 20 /$ and lately by Mourey et al. to examine the single crystal surface of $\mathrm{Zn} / 21 /$. However the main interest in these investigations was the lattice dynamical characteristics of surfaces and no report has been published yet concerning a ferromagnetic metal surface with vacuum deposited ${ }^{57} \mathrm{Co}$ as a source. Although there are some experimental difficulties, ${ }^{57} \mathrm{Co}$ source spectroscopy using UHV deposition is a unique technique to study surface magnetic properties of bulk single crystals. 
2.2. Absorbers with enriched surfaces.- In order to enhance the surface features in the Mössbauer absorption spectra, it is useful to enrich selectively the surfaces with ${ }^{57} \mathrm{Fe}$. Van der Kraan firstly tried to coat fine particles of $\alpha-\mathrm{Fe}_{2} \mathrm{O}_{3}$ with thin ${ }^{57} \mathrm{Fe}_{2} \mathrm{O}_{3}$ layers $/ 22 /$ and recently Morrish et al. attempted to prepare ${ }^{57} \mathrm{Fe}_{2} \mathrm{O}_{3}$-coated $\gamma-\mathrm{Fe}_{2} \mathrm{O}_{3} \mathrm{l} / 23 /$. Lauer et al, adopted this idea for UHV deposited thin films. They prepared, for instance, a film consisting of $\mathrm{Cu}, 100 \AA$ natural $\mathrm{Fe}, 5 \AA^{57} \mathrm{Fe}$ and $\mathrm{Cu}$ layers $/ 24 \%$. The hyperfine field at $4.2 \mathrm{~K}$ of the surface fraction was determined to be about $290 \mathrm{kOe}$, which is $15 \%$ smaller than the bulk.

If the isotope ${ }^{56} \mathrm{Fe}$ is used as the base, instead of natural $\mathrm{Fe}$, the surface enrichment in ${ }^{57} \mathrm{Fe}$ becomes more effective. The author's group prepared some UHV deposited films composed of ${ }^{56} \mathrm{Fe}$ and ${ }^{57} \mathrm{Fe}$ layers. The structures of the multilayer films are schematically shown in figure $2 a$. The surface was coated by $\mathrm{Sb}$ in one case and by $\mathrm{MgF}_{2}$ in the other case. An Fe film with the thickness of $100 \AA$ exhibits nearly bulk magnetic properties $/ 25,26 /$. The temperature of the substrate during the deposition was low enough to prevent diffusion, thus, most of ${ }^{57} \mathrm{Fe}$ nuclei would be located in the surface layers and the Mössbauer spectra are expected to show the surface behavior of a rather thick film.

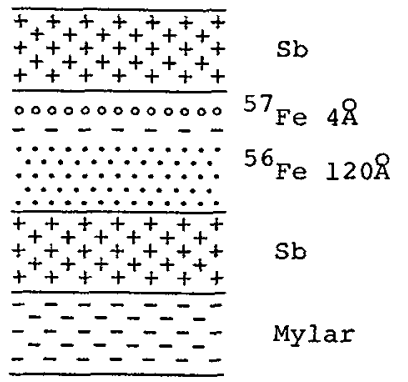

SAMPLE-I

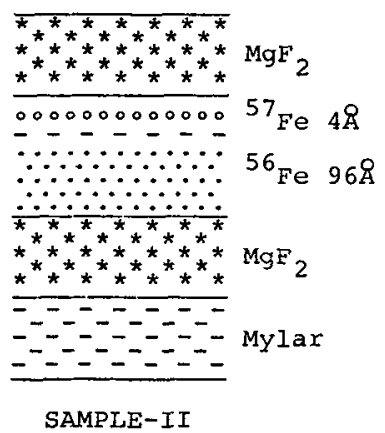

SAMPLE-II
Fig. 2a : Structure of the multilayer films. Sb and $\mathrm{MgF}_{2}$ layers are thicker than $200 \AA$.

Figure 2b shows the Mössbauer absorption spectra at $4.2 \mathrm{~K}$, with and without an external field.

The spectra without external fields consist of six-Iine patterns with intensity ratios of near1y $3: 4: 1: 1: 4: 3$, indicating the spontaneous magnetization of the surface layers are parallel to the surface plane. When an external field of $45 \mathrm{kOe}$ was applied perpendicularly to the surface (parallel to the gamma ray propagation), the second and fifth lines disappeared.

This fact means that the spins were oriented comple- tely along the magnetic field, indicating again that the samples are ferromagnetic and no dead layer exists in the interfaces.

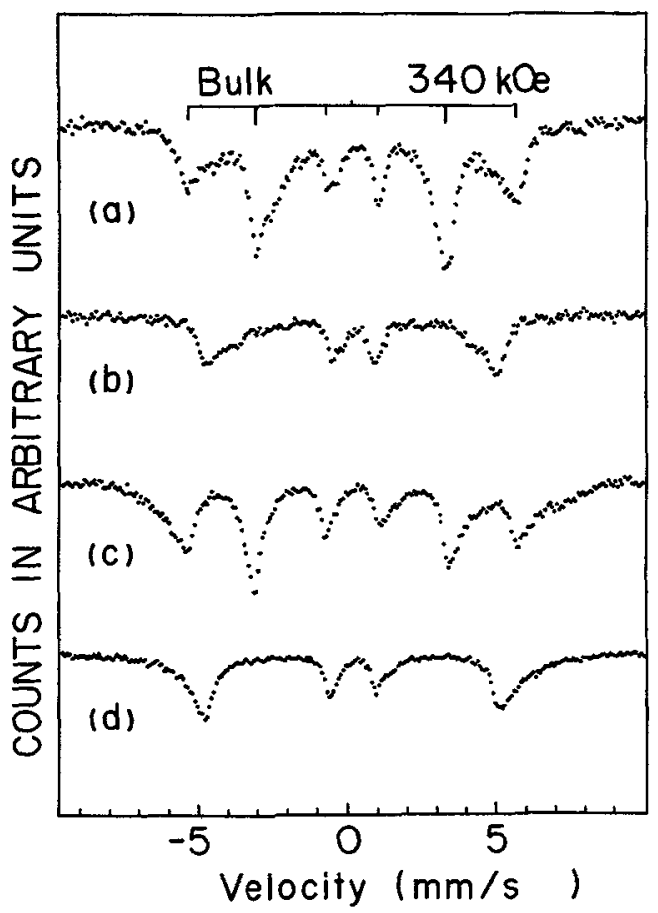

Fig. 2b : Mössbauer absorption spectra at $4.2 \mathrm{~K}$, a) $\mathrm{Sb}$-coated $\mathrm{Fe}$ surface, no external field;

b) $\mathrm{Sb}$-coated $\mathrm{Fe}$ surface with $45 \mathrm{kOe}$;

c) $\mathrm{MgF}_{2}$-coated $\mathrm{Fe}$ surface, no external field;

c) $\mathrm{MgF}_{2}$-coated $\mathrm{Fe}$ surface with $45 \mathrm{kOe}$.

By the external field, the line shapes of the first and the sixth lines were not greatly changed. The line broadening is mainly due to the distribution of the hyperfine field. The determination of the hyperfine field distribution by computor fitting is not yet completed but visually it appears that the surface effects in the two cases act in the opposite directions : reduction of the hyperfine field in the sb-coated case and increase of the hyperfine field in the $\mathrm{MgF}_{2}$-coated case.

Such an increase of the hyperfine field at the surface was also detected in the cases of Ag-coated surface $/ 27 /$, MgO-coated surface /28/ and granular $\mathrm{Fe}^{-\mathrm{SiO}_{2}}$ films /29/. On the other hand the decrease of the surface hyperfine field was found in the electrodeposited surfaces $/ 18,19 /$ and Cu-coated surface $/ 24 /$. It seems that the surface magnetic moment is not always smaller but is sometimes bigger than the bulk's moment. The surface magnetic effect is complicated and probably not explained by a simple mechanism.

2.3. Thin_fifilms.- Some problems in surface magne- 
tism can only be studied by using thin film samples. An example is the surface anisotropy whose effect is negligible in thick films. It is well known that the average orientation of the magnetic spins is determined by relative intensity ratio of the spectrum. Interesting results were obtained with MgO-coated thin Fe films $/ 28 /$. For thick films an intensity ratio of $3: 4: 1: 1: 4: 3$ has been found. In contrast, the thinnest film ( $8 \AA$ in the average) showed a drastically different ratio, being approximately $3: 1: 1: 1: 1: 3$. Thus the spontaneous magnetization changed to a preferential orientation along the normal to the surface. This is a clear evidence of the existence of surface anisotropy. Probably the surface anisotropy depends on the crystallographic orientation, the flatness of the surface, the nature of the coating material... Measurements on well defined surfaces as a function of thickness and also as a function of external fields will be necessary for a deeper understanding of surface anisotropy.

For a study of Ni surfaces, the author's group prepared thin $\mathrm{Ni}$ films including $5 \%{ }^{57} \mathrm{Fe}$, sandwiched between $\mathrm{MgF}_{2}$ layers /25/. The Mössbauer spectra are reproduced in figure 3.

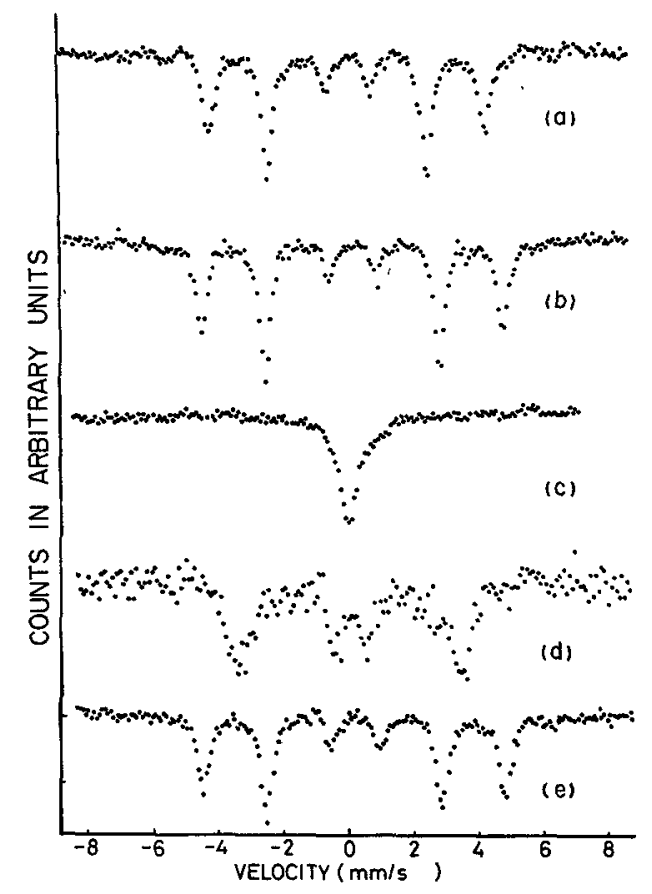

Fig. 3 : Mössbauer absorption spectra for the $\mathrm{MgF}_{2}-$ coated thin films of $\mathrm{Ni}\left(5 \%{ }^{57} \mathrm{Fe}\right)$,

a) $48 \AA-f i l m$ at $300 \mathrm{~K}-$ b) $48 \AA$-film at $4.2 \mathrm{~K}$ -

c) $16 \AA$-film at $300 \mathrm{~K}-$ d) $16 \AA$-film at $300 \mathrm{~K}$ with $7 \mathrm{kOe}-\mathrm{e}) 16 \AA-\mathrm{fi} 1 \mathrm{~m}$ at $4.2 \mathrm{~K}$.

In the case of a $48 \AA \mathrm{film}$, the absorption lines are sharp and the hyperfine field is equal to the bulk value, $263 \mathrm{kOe}$ at $300 \mathrm{~K}$ and $285 \mathrm{kOe}$ at $4.2 \mathrm{~K}$.
The intensity ratio is close to $3: 4: 1: 1: 4: 3$. In the case of a $16 \AA-f i l m$, a considerable fraction of $\mathrm{Fe}$ is close to the surface and the ${ }^{57} \mathrm{Fe}$ nuclei act as probes to surface anomalies, if any, such as dead layers or antiferromagnetic surface states. The spectrum at $4.2 \mathrm{~K}$ however is rather sharp and the hyperfine field is the same as the bulk within the experimental error. Surface anomaly could not be detected. The magnetic moment of $\mathrm{Fe}$ at the $\mathrm{Ni}-\mathrm{MgF}_{2}$ interface seems to be nearly the same as that in bulk $\mathrm{Ni}$ metal.

At $300 \mathrm{~K}$, the $16 \AA$-film behaves superparamagnetically. When an external field ( $7 \mathrm{kOe}$ ) was applied longitudinally, an effective hyperfine field of about $220 \mathrm{kOe}$ was induced. Therefore the intrinsic Curie temperature is much higher than $300 \mathrm{~K}$. The temperature dependence of thin film magnetization is only partially measurable.

Surfaces of non-metallic compounds were studied by using ultrafine particles $/ 22,23,30,31 /$. The surface anisotropy due to the coating by organic substances was discussed in the case of $\mathrm{NiFe}_{2} \mathrm{O}_{4}$ fine particles $/ 31$. It was claimed that the surface spin is canting in the case of $\gamma-\mathrm{Fe}_{2} \mathrm{O}_{3}$ fine particles $/ 23,30 /$.

2.4. Converersinion_elelectr. conversion electron Mössbauer measurements in a backscattering geometry are popularly used. This method is certainly suitable for corrosion and catalysis studies. A gas-flow type counter for this purpose is commercially available. The depth of the observation, however, extends over some $100 \AA$, which is too large to catch surface phenomena just near the top surface layer. Magnetic behaviors on nonmagnetic substances may be detected by using surface-enriched samples. The sensitivity was assured to be high enough for the detection of monolayers $132 /$.

Depth selective measurements were at tempted by using B-spectrometers to analyse the energy of electrons $133,34 /$. It was assumed that the energy loss of the conversion electron is proportional to the escape length, i.e. the depth from the surface. The resolution in thickness is in the order of $50 \AA$, which is not yet sufficient to observe magnetic surface anomalies.

The detection systems for conversion electrons are in progress, for example, Massenet and Daver succeeded to measure at liquid helium temperature using a channeltron /35/. The combination of surfaceenriched samples with improved detection techniques 
will be one possibility to study clean vacuum surfaces.

3. Remarks.- The advantages of Mössbauer spectroscopy in the investigation of surface magnetism will be summarized as follows;

(1) Surfaces and also interfaces of bulk crystals can be studied.

(2) Standard values of bulk crystals are well known.

(3) The orientation of the spins are determined by the relative intensity ratio of the hyperfine pattern.

(4) The chemical state of surface atoms is indicated by the spectra. The contamination by 0 , also by $\mathrm{C}$ or by $\mathrm{N}$ is distinguishable /36,37/.

Disadvantages are : the number of nuclear species is limited and Mössbauer measurements need relatively long times, therefore, it is difficult to keep a metallic surface clean.

Acknowledgements.- The author thanks Professor T. Takada for encouragements and Mr $\mathrm{S}$. Hine for collaborations. Thanks are also due to Professor T. Murao for discussion and Professor U. Gonser for critical reading of the manuscript.

This work was supported by the Grant for Fundamental Research in Chemistry.

\section{References}

/1/ Liebermann, L.N., Fredkin, D.R. and Shore, H.B. Phys. Rev. Lett. 22 (1969) 539.

12/ Liebermann, L.N., Clinton, J., Edwards, D.M. and Mathon, J., Phys. Rev. Lett. 25 (1970) 232.

13/ See a review by Gradmann, U., J. Mag. Mag.Mater. 6 (1977) 173. Original reports are cited there as references.

14/ Koepke,R. and Bergmann,G., Z.Phys.B21 (1975) 185.

15/ Sato,M. and Abe,K., Solid State Commun.26 (1978) 95. and Sato,M., Abe, K. and Endoh,Y., this conference.

/6/ See a review by Siegmann,H.C., Phys. Rep. 17C (1975) 38.

17/ Eicher, S., Rau, C. and Sizmann, R., J. Mag. Mag. Mater. 6 (1977) 204.

18/ Liebsch,A., Levin, K. and Bennemann, K.H., Solid State Commun. 13 (1973) 347.

19/ Fulde,P., Luther,A. and Watson,R.E., Phys. Rev. B8 (1973) 440 .

/10/ Desjonquèrers,M.C. and Cyrot-Lackmann, F.,Solid State Commun. 20 (1976) 855.

/11/ Teraoka,Y. and Kanamori,J., private communication.
/12/ Allan,G. Surf.Sci. 74 (1978) 79.

/13/ Zhang, H.I., Phys. Rev. B16 (1977) 3194.

/14/ Binder, K. and Hohenberg, P.C., Phys. Rev. B9 (1974) 2194.

/15/ Palmberg, P.W., DeWames, R.E. and Vredevoe,L.A. Phys. Rev. Lett. 21 (1968) 682 .

/16/ Namikawa, K., J. Phys. Soc. Japan 44 (1978) 165.

/17/ Takayama,H., Bohnen, K., -P. and Fulde, P., Phys. Rev. B14 (1976) 2287.

/18/ Shinjo, T., Matsuzawa, T., Mizutani, T. and Takada, T., Japan. J.App1. Phys.sup.2, pt.2 (1974) 729 .

/19/ Shinjo, T., Matsuzawa, T., Takada, T., Nasu,S. and Murakami, Y., J. Phys Soc. Japan 32 (1973) 1032.

/20/ Burton,J.W. and Godwin,R.P., Phys. Rev. 158 (1967) 218 .

121/ Mourey, C1., Marcha1,G. and Janot, Chr., J. Physique Colloq. 37 (1976) C6-713.

122/ Van der Kraan,A.M., Phys. Status Solidi a 18 (1973) 215.

/23/ Morrish,A.H., Haneda, K. and Schurer, P.J., J. Physique 37 (1976) C6-30l. and Haneda, K. and Morrish, A.H., Phys. Lett. 64A (1977) 259.

124/ Lauer, J., Keune, W. and Shinjo, T., Physica 86-88B (1977) 1409.

/25/ Shinjo,T., Hine,S. and Takada,T., Proc. 7th Intern. Vac. Congr. \& 3rd Intern. Conf. Solid Surfaces (Vienna 1977), p.2655.

126/ Hine,S., Shigematsu, T., Shinjo, T. and Takada $T$., this conference.

127/ Duncan, S., Semper, R., Chien, C.L. and Walker, J.C., presented at International Conference on Mössbauer Spectroscopy, Bucharest, 1977 and Walker, J.C., Owens, A.H. and Chien, C.L., this conference.

/28/ Shinjo, T., Hine, S. and Takada, T., this conference.

/29/ Dormann,J. -L., Gibart, P. and Renaudin, P., J. Physique Co11oq. 37 (1976) C6-281.

/30/ Coey, J.M.D. and Khalafalia, D., Phys. Status Solidi a 11 (1972) 229.

/31/ Berkowitz, A.E., Lahut, J.A., Jacobs, I.S., Levinson, M. and Forester, D.W., Phys. Rev. Lett. 34 (1975) 594.

132/ Petrera, M., Gonser, U., Hasmann, U., Keune. W. and Lauer, J., J. Physique Colloq. 37 (1976) C6-295.

/33/ Bäverstam, U., Ekdah1, T., Bohm, C., Liljequist, D. and Lingström, B., "Mössbauer Effect Methodology, vo1. 9" ed. by Seide1, C. and Gruverman, I.J., (P1enum, N.Y.) 1974, p. 259.

134/ Toriyama, T., Kigawa, M., Fujioka, M. and Hisatake, K., Japan. J. Appl. Phys. sup. 2, pt. 1 (1974) 733.

135/ Massenet, O., and Daver, H., Solid State Commun. 25 (1978) 917.

/36/ Goodwin, J.G. and Parravano, G., J. Vac. Sci. Techno1., 14 (1977) 1157.

/37/ Longworth, G. and Hartley, N.E.W., Thin Solid Films 48 (1978) 95. 